\title{
Hemifacial microsomia
}

\author{
Sonali Ullal · Ajit Mahale · Kalyan Paudel
}

\begin{abstract}
Hemifacial microsomia (HFM) is a craniofacial disorder characterized by a wide spectrum of anomalies, including conductive hearing loss due to external and middle ear deformities [1]. Hemifacial microsomia is the second most common developmental craniofacial anomaly after cleft lip and palate. Diagnostic imaging is important for presurgical evaluation of patients with this anomaly; however the broad spectrum of abnormalies encountered in patients with hemifacial microsomia can be confusing [2]. We present a case of a hemifacial microsomia in 12 year old male.
\end{abstract}

Keywords Hemifacial · Microsomia $\cdot$ OMENS - Clas sification

S. Ullal · A. Mahale · K. Paudel

Deptartment of Radiodiagnosis,

Kasturba Medical College Hospital, Attawar, Mangalore - 575 001, India

K. Paudel ( $₫)$

E mail: paude16@gmail.com

\section{Case report}

A 12-year-old male presented to our hospital with asymmetry of the lower half of his face. He did not give history of significant hearing loss. He had history of absence of anal orifice at birth for which he underwent colostomy followed by perineal rectoplasty. His siblings are completely healthy. His physical examination revealed well built and moderately nourished male with normal vital signs. He showed a remarkable facial hypoplasia on the left side with the chin deviating to the left. Preauricular tags (Fig. 1) were present at the site of left ear and external auditory canal and middle ear were not present on the same side. On palpation, ramus of mandible was smaller on left side as compared to right and premolars had not erupted. Weber test (conductive hearing test) was lateralized to the left. Computed tomography (CT) was performed to include head, temporal bone and neck region. Three dimensional images were reformatted for detail evaluation of the osseous abnormalities. On the affected side (Fig. 2) mandible was hypoplastic, malformed and articulated with the glenoid fossa. The zygomatic arch was incomplete, maxilla and squamous temporal bone were small and there was bony atresia of external auditory canal. On the right side mandible, maxilla, zygomatic arch, squamous temporal bone, external auditory canal and temporo-mandibular joint were normal. Lateral pterygoid muscle on the left side was small and poorly formed (Fig. 3) as compared to the right side.

\section{Discussion}

Hemifacial microsomia( HFM) is a congenital condition in which the lower half of the face is unilaterally under-developed and does not catch up with normal growth during childhood. The occurrence of the hemifacial microsomia is between 1 in 3000 and 1 in 5600 births. Males are more frequently affected than females and the right side is affected more often then the left [3]. However, in our patient the left side was affected. 


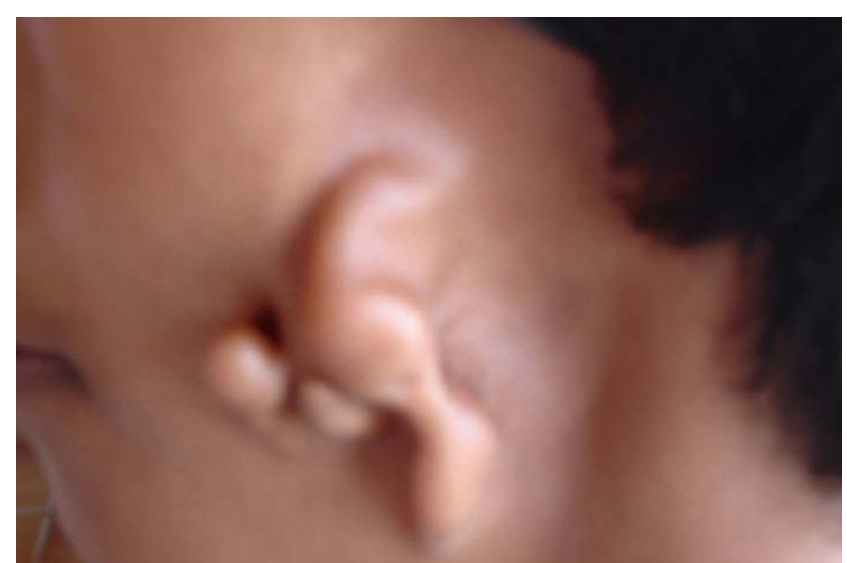

Fig. 1 12-year-old boy with left hemifacial microsomia showing auricular tags on affected side

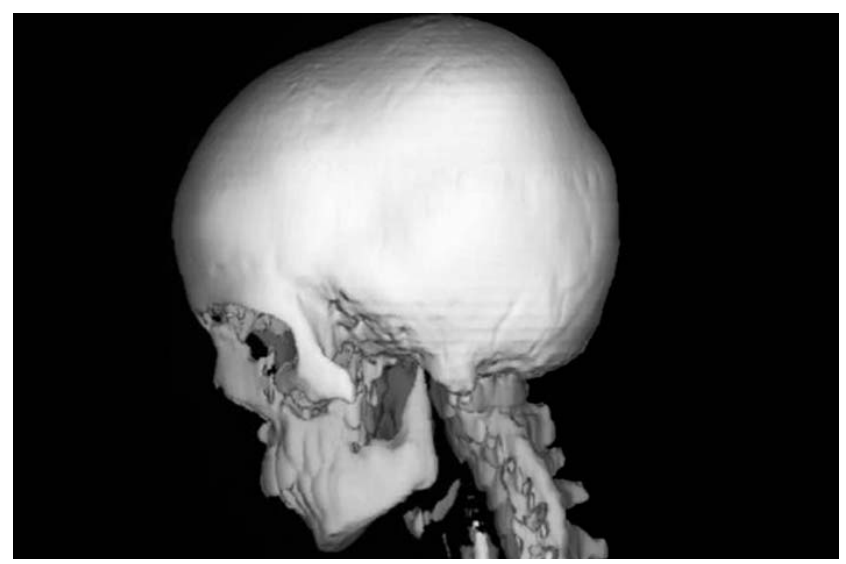

Fig. 2 Left hemifacial microsomia: Three-dimensional CT reconstruction shows hypoplastic and malformed left mandible, incomplete zygomatic arch, small maxilla and squamous temporal bone and bony atresia of external auditory canal

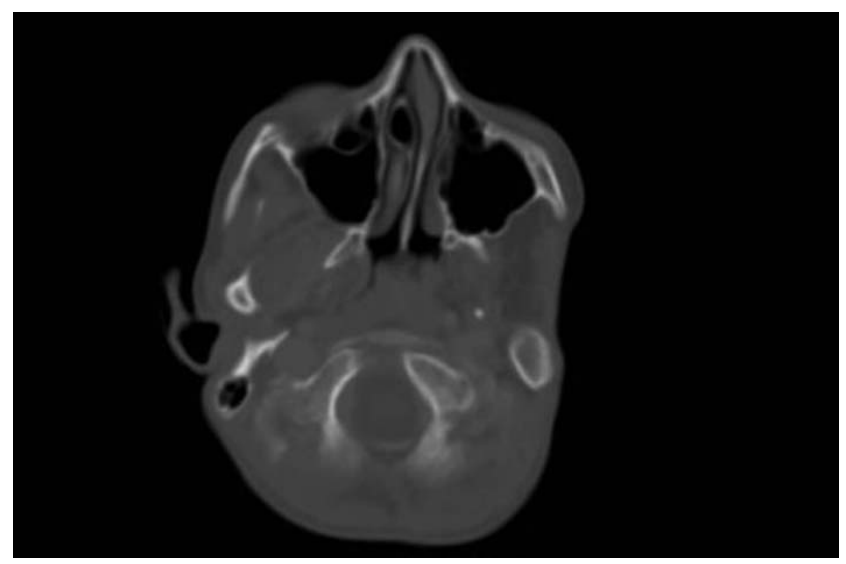

Fig. 3 CT scan shows normal right lateral pterygoid muscle inserting along lateral surface of lateral pterygoid plate. On the left, small and poorly formed lateral pterygoid muscle
HFM primarily is a syndrome of the first branchial arch, involving underdevelopment of the temporomandibular joint, mandibular ramus, muscles of mastication and the ear. Abnormal development of the auricular hillocks leads to microtia or atresia of the pinna and is proportional in severity to the abnormal external auditory canal development [2]. Abnormal accessory hillocks become preauricular tags, which were present in our patient. Hearing loss may results from underdevelopment of the osseous components of the auditory system and a diminished or absent external auditory meatus. Occasionally second branchial arch defects involving the facial nerve and facial muscles coexist with hemifacial microsomia [5].

More than 15 terms, including Goldenhar's syndrome and oculoauriculovertebral dysplasia, have been applied to this disease, with each term representing the perspectives of different specialists [2]. The two most frequently used classifications are the skeletal-auricular-soft tissue (SAT) and the orbital asymmetry-mandibular hypoplasia-ear malformation-nerve dysfunction-soft tissue deficiency (OMENS) classification [3]. The OMENS classification is the most comprehensive and, therefore, one of the most commonly used systems [5]. In our patient orbital involvement and facial nerve dysfunction were absent.

In normal growth, forces exerted by the developing muscles of mastication contribute to the formation of the facial skeleton [5]. In patients who have hemifacial microsomia, the associated muscles may be smaller and underveloped, thereby adversely affecting the maturation of facial bones [6]. In more severe cases, entire portions of the mandible fail to develop and thus accentuate the clinical presentation. The combined deficiency of the soft tissue and osseous structures of the maxillofacial region result in the clinical manifestations characteristic of HFM.

Computed tomography (CT) can provide both a three dimensional rendition of the soft tissue of the face and an image of the underlying bone. It helps to establish the degree of anatomical malformation and the relationship of the deformity to the adjacent craniofacial skeleton.

The management of HFM exemplifies the need for an interdisciplinary approach to craniofacial anomalies. There are essentially two approaches: either an early (during growth) or a late (after the active growth period) surgical intervention [3].

When treating the asymmetry with a costo-chondral bone graft, the goal is to replace the distorted or even absent condyle with a new growth centre. This will only give the desired result when there is still some growth left [3]. A costo-chondral graft provides length to the ramus and it also acts as a growth centre. For most children, a single operation is sufficient to correct the asymmetry.

The other procedures to correct hemifacial microsomia include classical osteotomy or a distraction with a surgical intervention. Distraction osteogenesis is increasingly advocated in treating patients with HFM as it is considered a 
good alternative to the classical surgical interventions (like osteotomies and bone grafts) and its presumed positive effect on the soft tissue as claimed by the advocators [3].

\section{Summary}

Hemifacial microsomia (HFM) is a variable asymmetric craniofacial malformation resulting in hypoplasia of the components of the first and second branchial arches. The use of the OMENS classification system, complete audiologic evaluation and temporal bone computed tomography is useful to evalauate the condition. The management of HFM is a multidisciplinary approach which needs to be individually customized.

\section{References}

1. Carvalho Gerald J, Song Caroline S, Vargervik Karin, Lalwani Anil K (1999) Auditory and facial nerve dysfunction in patients with hemifacial microsomia. Arch Otolaryngol Head Neck Surg 125:209-212

2. Sze Raymond W, Paladin Angelisa M, Lee Samson, Cunningham Michael (2002) Hemifacial microsomia in pediatrics patients: asymmetric abnormal development of the first and second brachial arches. AJR 178: $1523-1530$

3. Romsee MC, Verdonck A, Schoenaers J, Careals C (2004) Treatment of hemifacial microsomia in a growing child: the importance of co-operation between the orthodontist and the maxillofacial surgeon. J Orthod 31: 190-200

4. Rahbar R, Robson CD, Mulliken JB et al (2001) Craniofacial, temporal bone and audiologic abnormalities in the spectrum of hemifacial microsomia. Arch Otolaryngol Head Neck Surg 127:265-271

5. Monahan R, Seder K, Patel P, Alder M, Grud S, Gara M (2001) Hemifacial microsomia: etiology, diagnosis and treatment. J Am Dent Assoc 132:1402-1408

6. Murray JE, Kaban LB, Mulliken JB (1984) Analysis and treatment of hemifacial microsomia. Plast Reconstr Surg 74: 186-199 